\title{
THE ORTHOGONAL INVARIANTS OF A CURVE IN HILBERT SPACE ${ }^{1}$ \\ F. ALBERTO GRÜNBAUM
}

\begin{abstract}
Let $x(t), y(t)(t \in R)$ be a pair of continuous curves in $H$, not passing through the origin. We consider the problem of deciding when one curve is obtained by rotating the other one.
\end{abstract}

The problem of orthogonal invariants for $n$ vectors $x_{1}, \cdots, x_{n}$ in Hilbert space is a classical one. The matrix of all the scalar products

$$
\left\|\left\langle x_{i}, x_{j}\right\rangle\right\|
$$

is a complete table of invariants. See [1, p. 32].

Here we consider the problem of a continuous curve in Hilbert space and ask for its orthogonal invariants. The result is that the determinants of matrices like (1) are already a complete list of invariants.

More precisely, if we denote by $H(z)$ the closed linear span of a curve $z(t)$ in Hilbert space, we can formulate

THEOREM. Let $x(t)$ and $y(t)$ be a pair of continuous curves in Hilbert space. Assume that neither of them passes through the origin. Assume that for any choice of $t_{1}, \cdots, t_{n}\left(t_{i} \in R, n \geqq 1\right)$ the relation

$$
\operatorname{det}\left\|\left\langle x\left(t_{i}\right), x\left(t_{j}\right)\right\rangle\right\|=\operatorname{det}\left\|\left\langle y\left(t_{i}\right), y\left(t_{j}\right)\right\rangle\right\|
$$

is satisfied.

Then there exists a partial isometry $V$ in Hilbert space, mapping $H(x)$ onto $H(y)$, such that

$$
y(t)=V x(t) \text { for all } t \in R .
$$

Remark I. Condition (2) can be written as

$$
\left|x\left(t_{1}\right) \wedge \cdots \wedge x\left(t_{n}\right)\right|=\left|y\left(t_{1}\right) \wedge \cdots \wedge y\left(t_{n}\right)\right| .
$$

Now, if the curves are infinitely smooth we can deduce from $\left(2^{\prime}\right)$

$$
\left|x^{(m)}(t) \wedge \cdots \wedge x^{(1)}(t)\right|=\left|y^{(m)}(t) \wedge \cdots \wedge y^{(1)}(t)\right| \quad \text { for all } t \in R ;
$$

Received by the editors November 3, 1972.

AMS (MOS) subject classifications (1970). Primary 53A99, 53C99.

1 This work was supported by U.S. Air Force Grant AFOSR-71-2053.

(c) American Mathematical Society 1974 
where the superscript denotes differentiation. In this form our statements look a bit closer to known results in differential geometry. See for instance $[2$, p. 160]. However, in the absence of smoothness conditions (2) cannot be localized.

REMARK II. That $x(t)$ be different from the vector zero and continuous are clearly necessary conditions. Indeed the point of the theorem is that the continuity assumption makes (2) into a very strong condition: The equality of all these determinants forces the equality of the corresponding matrices.

The Theorem is an easy consequence of the following two lemmas.

LEMMA 1. Given all the determinants (2) one can determine each one of the expressions

$$
\sum_{\sigma}\left\langle x\left(t_{1}\right), x\left(t_{\sigma 1}\right)\right\rangle \cdots\left\langle x\left(t_{n}\right), x\left(t_{\sigma n}\right)\right\rangle,
$$

the sum extended over all cycles in the $\left(t_{1} \cdots t_{n}\right)$.

Proof. Label each term entering in the determinant according to the corresponding permutation. Now group these terms as follows: first all those corresponding to cycles, then those corresponding to permutations which factor into two cycles, and keep going according to the number of factors.

Notice that the signature of any cycle of length $m$ is $(-1)^{m-1}$. Using this last remark one proves by induction on $n$ that the information contained in the family of determinants $\operatorname{det}\left\langle x\left(t_{i}\right), x\left(t_{j}\right)\right\rangle, t_{1} \cdots t_{n} \in R$, is equivalent to that contained in the family of expressions (3).

LEMMA 2. The knowledge of all the products

$$
\sum_{\sigma}\left\langle x\left(t_{1}\right), x\left(t_{\sigma 1}\right)\right\rangle \cdots\left\langle x\left(t_{n}\right), x\left(t_{\sigma n}\right)\right\rangle
$$

determines $\langle x(t), x(s)\rangle \forall(t, s)$.

Proof. Notice that (3) can be written as (proportional to)

$$
\sum_{\pi}\left\langle x\left(t_{\pi 1}\right), x\left(t_{\pi 2}\right)\right\rangle\left\langle x\left(t_{\pi 2}\right), x\left(t_{\pi 3}\right\rangle \cdots\left\langle x\left(t_{\pi n}\right), x\left(t_{\pi 1}\right)\right\rangle\right.
$$

where $\pi$ ranges over all permutations of the $n$ symbols $\left(t_{1} \cdots t_{n}\right)$ : At this point we can refer the reader to [3], where a complete proof of the lemma is carried out for a different purpose. We include here an outline containing all the main ideas of that proof.

Using the information contained in (4) for $n=2$ one gets $\langle x(t), x(s)\rangle^{2}$. Now, for $(t, s)$ close enough to the diagonal $(t=s)$ this is all that is needed because $\langle x(t), x(s)\rangle$ is continuous and nonzero for $t=s$. 
For $(t, s)$ away from the diagonal one shows that $\langle x(t), x(s)\rangle$ being undecidable on the basis of (4) implies that

$$
\left\langle x(t), x\left(e_{1}\right)\right\rangle\left\langle x\left(e_{1}\right), x\left(e_{2}\right)\right\rangle \cdots\left\langle x\left(e_{n}\right), x(s)\right\rangle=0
$$

for any choice of $e_{1} \cdots e_{n}$ satisfying $t<e_{1}<\cdots<e_{n}<s$. This clearly gives a contradiction.

Relation (5) is proved by induction on $n$. For $n=1$ we use the expression (4) setting

$$
t_{1}=t, \quad t_{2}=e_{1}, \quad t_{3}=s .
$$

We conclude that

$$
\left\langle x(t), x\left(e_{1}\right)\right\rangle\left\langle x\left(e_{1}\right), x(s)\right\rangle\langle x(s), x(t)\rangle
$$

is known, and because $t\left\langle e_{1}<s\right.$ we can assume that $\left\langle x(t), \dot{x}\left(e_{1}\right)\right\rangle$ and $\left\langle x\left(e_{1}\right), x(s)\right\rangle$ are known. Then their product has to be zero or else $\langle x(t), x(s)\rangle$ would be known. This proves (5) for $n=1$. The boundary behavior of $e_{1}$ should be carefully handled, see [3].

For $n=2$ take $e_{1}, e_{2}$ such that $t<e_{1}<e_{2}<s$. Set

$$
t_{1}=t, \quad t_{2}=e_{1}, \quad t_{3}=e_{2}, \quad t_{4}=s .
$$

With this choice of $t$ 's we write (4) in the form

$$
\begin{aligned}
\langle x(t), x(s)\rangle\left[\left\langle x(t), x\left(e_{1}\right)\right\rangle\left\langle x\left(e_{1}\right), x\left(e_{2}\right)\right\rangle\left\langle x\left(e_{2}\right), x(s)\right\rangle\right. \\
\left.+\left\langle x(t), x\left(e_{2}\right)\right\rangle\left\langle x\left(e_{2}\right), x\left(e_{1}\right)\right\rangle\left\langle x\left(e_{1}\right), x(s)\right\rangle\right]+\cdots .
\end{aligned}
$$

The terms not explicitly written out do not involve $\langle x(t), x(s)\rangle$ and thus are known.

The coefficient of $\langle x(t), x(s)\rangle$ has to be zero by hypothesis. We will see now that its first term (which is of the form (5)) has to be zero. We do this by showing that any term that is not the first one has to vanish. In fact multiplying and dividing by $\left\langle x(t), x\left(e_{1}\right)\right\rangle$ we get

$$
\begin{aligned}
\left\langle x(t), x\left(e_{2}\right)\right\rangle\left\langle x\left(e_{2}\right), x\left(e_{1}\right)\right\rangle & \left\langle x\left(e_{1}\right), x(s)\right\rangle \\
= & \frac{\left\langle x(t), x\left(e_{2}\right)\right\rangle\left\langle x\left(e_{2}\right), x\left(e_{1}\right)\right\rangle}{\left\langle x(t), x\left(e_{1}\right)\right\rangle} \cdot\left\langle x(t), x\left(e_{1}\right)\right\rangle\left\langle x\left(e_{1}\right), x(s)\right\rangle .
\end{aligned}
$$

The second factor is of the form (5) for $n=1$ and thus vanishes. Notice that $\left\langle x(t), x\left(e_{1}\right)\right\rangle \neq 0$ or else we already had (5) for $n=2$.

In the general case the inductive hypothesis is carried out in a similar fashion. See [3]. The lemma is proved.

PROOF OF THE THEOREM. Now that we have

$$
\langle x(t), x(s)\rangle=\langle y(t), y(s)\rangle \quad \forall t, s
$$

one simply puts $x(t)$ in correspondence with $y(t)$, and tries to extend the 
map in a linear fashion. For that you need to satisfy the compatibility condition

$$
\sum a_{i} x\left(t_{i}\right)=0 \Leftrightarrow \sum a_{i} y\left(t_{i}\right)=0 .
$$

This and the possibility of extending the mapping to an isometry from $H(x)$ onto $H(y)$ follow immediately from (6).

In conclusion we notice that in general no bound can be put on the size of the determinants (2) if one wants to recover $\langle x(t), x(s)\rangle$.

\section{REFERENCES}

1. H. Weyl, The classical groups. Their invariants and representations, 2 nd ed., Princeton Univ. Press, Princeton, N.J., 1946.

2. H. Guggenheimer, Differential geometry, McGraw-Hill, New York, 1963. MR 27 \#6194.

3. F. Alberto Grünbaum, The square of a Gaussian process, Z. Wahrscheinlichkeitstheorie und Verw. Gebiete 23 (1972), 121-124.

Courant Institute of Mathematical Sciences, New York University, New YORK, NEW YORK 10012

Current address: Department of Applied Mathematics, California Institute of Technology, Pasadena, California 91109 